\title{
Modulation of endothelial cell proliferation and capillary network formation by the ox-LDL component: 1-palmitoyl-2-archidonoyl- sn-glycero-3-phosphocholine (ox-PAPC)
}

\author{
B. Kiec-Wilk • A. Polus • U. Razny • \\ U. Cialowicz $\cdot$ A. Dembinska-Kiec
}

Received: 7 May 2010/Accepted: 28 December 2010/Published online: 15 January 2011

(C) Springer-Verlag 2011

\begin{abstract}
Atherosclerotic plaque formation is often associated with pathological angiogenesis. Modified phospholipids, including oxidized lipoproteins such as LDL, are found to induce adhesion of the monocytes to the endothelial cells and to stimulate their chemotaxis. Effects of oxidized 1-palmitoyl-2-archidonoyl-sn-glycero-3-phosphocholine (ox-PAPC) mimic actions of minimally modified LDL in vivo. Interleukin-8 (IL-8) and interleukin-15 (IL-15) are known to induce both inflammation and angiogenesis. The goal of our study was to analyze a potential synergism between ox-PAPC and IL-15 in the in vitro model of angiogenesis carried out in the human endothelial cells (HUVECs). Increasing IL-15 concentrations led to formation of the tube-like structures in the matrigel 3D-model of angiogenesis $(P<0.05)$, in contrast to ox-PAPC that inhibited this process. HUVECs incubation with ox-PAPC led to reduced IL-15 gene basal expression $(P=0.033)$ along with parallel increase, however statistically insignificant, of basal gene expression of IL-8 $(P=0.086)$. Our findings point to the ox-PAPC opposite effects on the IL-8- and IL-15-mediated angiogenic responses that contribute to pathological angiogenesis induced by ox-LDL.
\end{abstract}

Keywords Angiogenesis - Atherosclerosis - HUVEC . IL-8 · IL-15 · Ox-PAPC

B. Kiec-Wilk · A. Polus · U. Razny · U. Cialowicz ·

A. Dembinska-Kiec

Department of Clinical Biochemistry,

Jagiellonian University of Krakow, Krakow, Poland

B. Kiec-Wilk $(\bowtie)$

Department of Metabolic Disorders,

Jagiellonian University of Krakow,

Kopernika 15, 31-501 Krakow, Poland

e-mail: mbkiec@gmail.com

\section{Introduction}

Oxidized lipids promote cellular endoplasmic reticulum stress, autophagy, and chronic inflammatory response, all of which are observed in atherosclerosis [1]. One of these activated lipids, oxidized 1-palmitoyl-2-archidonoylsn-glycero-3-phosphocholine (ox-PAPC), mimics in vivo effects of an oxidized low-density lipoprotein, namely minimally modified LDL (mm-LDL) [2], and it induces expression of interleukin 8 (IL-8) in human endothelial cells [3].

Interleukin 15 (IL-15) is a cytokine-detected in the early phases of different chronic inflammatory processes [4]. Increased expression of IL-15 is observed in the atherosclerotic plaques, including endothelial cells [5]. Moreover, IL-15 is found to induce angiogenesis in vivo [6].

Since angiogenesis is observed in the majority of inflammatory processes, including atherosclerotic plaque formation, the goal of our study is to analyze activity of ox-PAPC in regulation of the IL-15 generation by endothelial cells and IL-15-mediated angiogenesis in the in vitro models of angiogenesis.

\section{Materials and methods}

Culture cell lines

Human umbilical venous endothelial cells (HUVEC) were isolated as described previously [7] and incubated with one of the following compounds: $75-150 \mu \mathrm{g} / \mathrm{ml}$ ox-PAPC (Cell Sciences), 0.2 nM VEGF (Sigma-Aldrich), 0.5 nM bFGF (Sigma-Aldrich) or $100 \mathrm{U} / \mathrm{ml} \mathrm{TNF} \alpha$ (Sigma-Aldrich) for 0-24 h. Any cytotoxic effects of the above factors were estimated by measurement of lactate dehydrogenase (LDH) 
activity in the medium (Cytotox 96 kit, Promega). Potential changes in cell proliferation were reflected by the rate of DNA synthesis, which was measured by bromo-deoxyuridine (BrdU) incorporation into DNA using the anti-BrdU assay (Roche Applied Science).

The IL-15 gene expression

Quantitative real-time PCR (qRT-PCR) was performed in order to investigate regulation of the gene expression, using GAPDH (GAPDH sense: gccagccgagccacatc; antisense: gcgcccaatacgaccaaa) as the reference gene. Total RNA was isolated by Trizol method (Invitrogen Life Technologies), and $1 \mu \mathrm{g}$ of the generated RNA was used to synthesize cDNA in the presence of SuperScript reverse transcriptase (Gibco). The qRT-PCR was performed in a reaction mixture containing QuantiTect SYBR Green PCR (Qiagen) mix and specific primers (IL-15 sense: ccgtggctttgagtaatga, and antisense: cagattctgttacattccc) according to the standard protocol of the Light Cycler system (Roche).

The 3-dimensional angiogenesis model in matrigel (the 3D matrigel model)

The HUVEC were re-suspended in matrigel (Calbiochem) $\left(10 \times 10^{6}\right.$ cells per $\left.1 \mathrm{ml}\right)$ and incubated with investigated factors for $24 \mathrm{~h}$. Number and length of vascular tubules were analyzed under optical microscope $(\times 10)$, photographed and analyzed by the computer software [7].

\section{Statistical analysis}

Descriptive results of continuous variables were expressed as mean values $\pm \mathrm{SD}$. Normal distribution and homogenity of variables were tested before in-depth statistical analysis. We used the $\chi^{2}$ test for comparisons of proportions and unpaired $t$ test for comparisons of the quantitative variables. Statistical significance was set at $P<0.05$. The statistical analysis was performed with Statistica for Windows from Statsoft.

\section{Results}

All of the investigated ox-PAPC $(150 \mu \mathrm{g} / \mathrm{ml})$ and IL-15 ( 2 and $8 \mathrm{nM}$ ) were safe under our experimental conditions, since they did not increase LDH concentration in tested HUVECs compared to the control, un-stimulated cells (Fig. 1). Physiological amounts of IL-15 (2, 4, and $8 \mathrm{nM})$ did not influence cell proliferation (EC) (Fig. 2). However, increasing IL-15 concentrations led to enhanced formation of the endothelial tubular-like structures, increased tubular

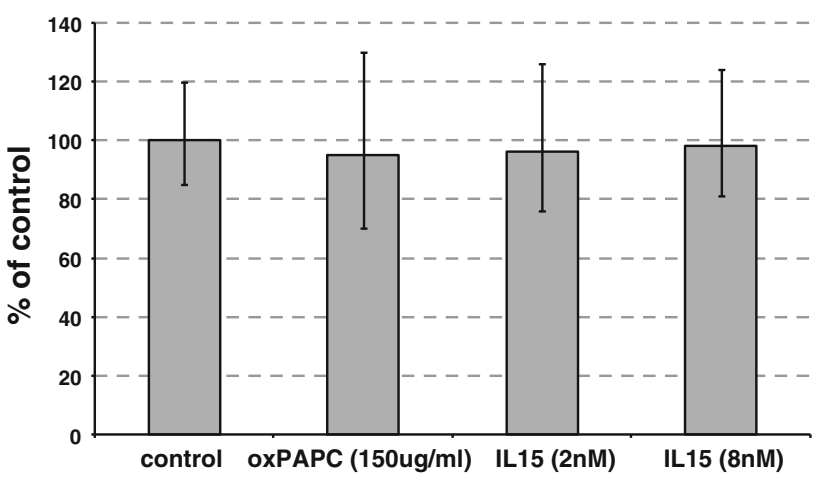

Fig. 1 Influence of investigated factors (ox-PAPC and IL-15) on cytotoxicity in HUVEC (measured as LDH production). The mean values $\pm \mathrm{SEM} ; n=3$ done in triplicates. VEGF-vascular endothelial growth factor, bFGF-basic fibroblast growth factor, IL-15interleukin 15, ox-PAPC-oxidized 1-palmitoyl-2-archidonoyl-snglycero-3-phosphocholine

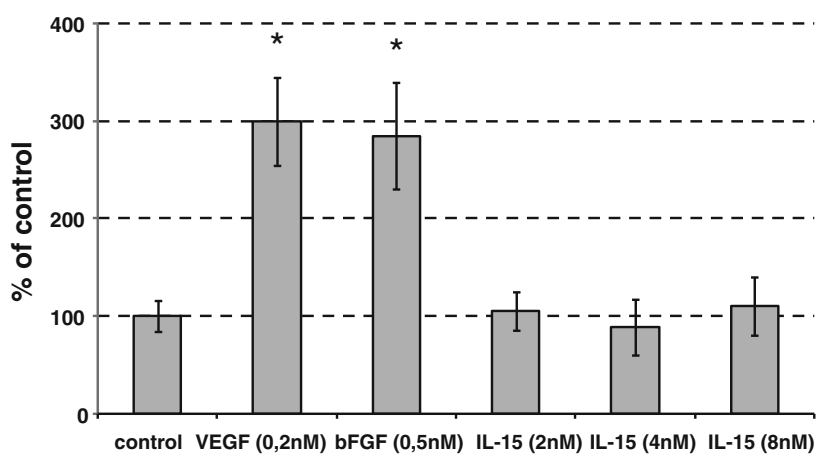

Fig. 2 Influence of IL-15 on HUVEC proliferation (measured as BrdU incorporation). Presented results are the mean values \pm SEM; $n=3$ done in triplicates. Incubation with VEGF and bFGF served as the positive controls. $P<0.005$ vs control. VEGF-vascular endothelial growth factor, bFGF-basic fibroblast growth factor, IL-15-interleukin 15

number and length in the 3D matrigel model in vitro $(P<0.05)$ (Figs. 3 and 4$)$. The observed angiogenic effect of $8 \mathrm{nM}$ IL-15 was comparable to the one of the pro-angiogenic growth factors such as VEGF and bFGF. Non-toxic amount of ox-PAPC $(75-150 \mu \mathrm{g} / \mathrm{ml})$, which cellular safety was verified by CytoTox screen (Fig. 1), did not influence the EC proliferation, but it inhibited the tubular-like endothelial structures formation in the 3D model (Fig. 4).

Next, we focused on the ox-PAPC effects over basal expression of the IL-15 gene in HUVECs. The timedependent inhibition of IL-15 expression in the endothelial cells was found after their incubation with $150 \mu \mathrm{g} / \mathrm{ml}$ ox-APC as well as $100 \mathrm{U} / \mathrm{ml} \mathrm{TNF} \alpha$, a known cellular endoplasmic reticulum stress-inducing factor (Fig. 5). The IL-15 gene inhibition was statistically significant after $3.5 \mathrm{~h}(P=0.033)$ or longer incubation time $(P=0.032$ at 


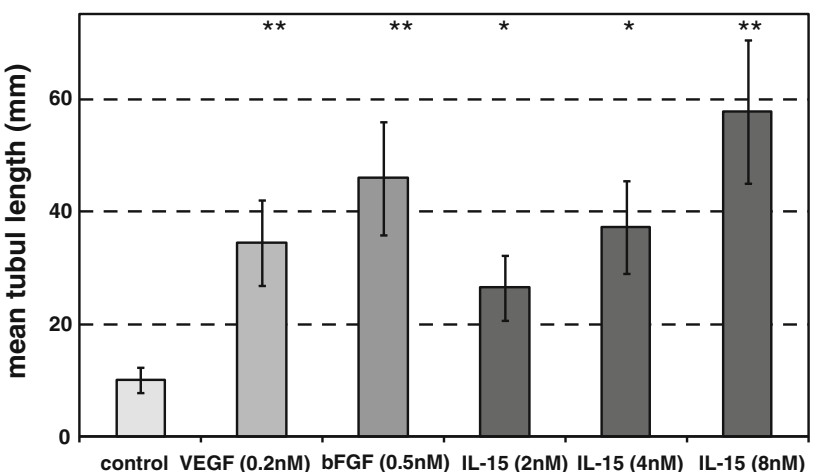

Fig. 3 IL-15-induced acceleration of the endothelial tube-like structure formation. The mean values $\pm \mathrm{SEM}$; significance $(* P<0.05$; $* * P<0.005)$ vs control, $n=3$ done in triplicates. Incubation with VEGF and bFGF served as the positive controls. VEGF-vascular endothelial growth factor, bFGF-basic fibroblast growth factor, IL-15-interleukin 15

$7 \mathrm{~h})$. We demonstrated that inhibition of the IL-15 gene expression was not a result of ox-PAPC toxic effect, since ox-PAPC at used concentrations did not stimulate the LDH release up to $24 \mathrm{~h}$ of incubation (Fig. 1). In the control, parallel experiment, there was a time-dependent, not statistically significant, up-regulation of the IL-8 gene expression induced by $150 \mu \mathrm{g} / \mathrm{ml}$ ox-PAPC $(P=0.086)$ (Fig. 6).

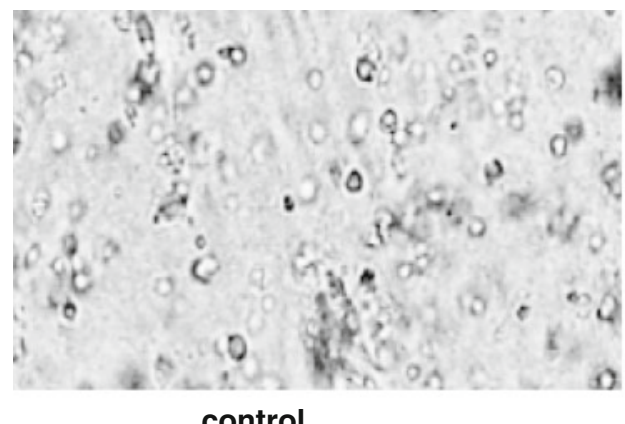

control

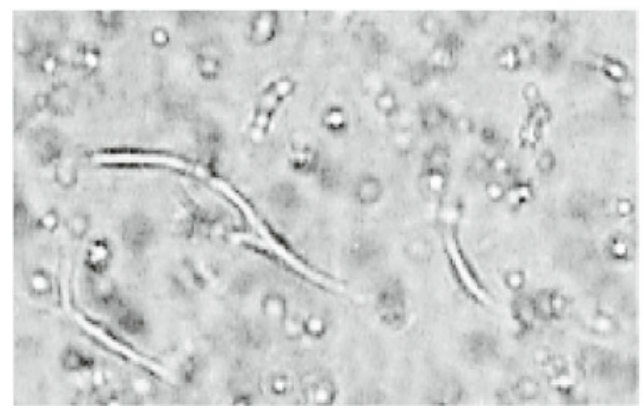

IL-15 (8nM)

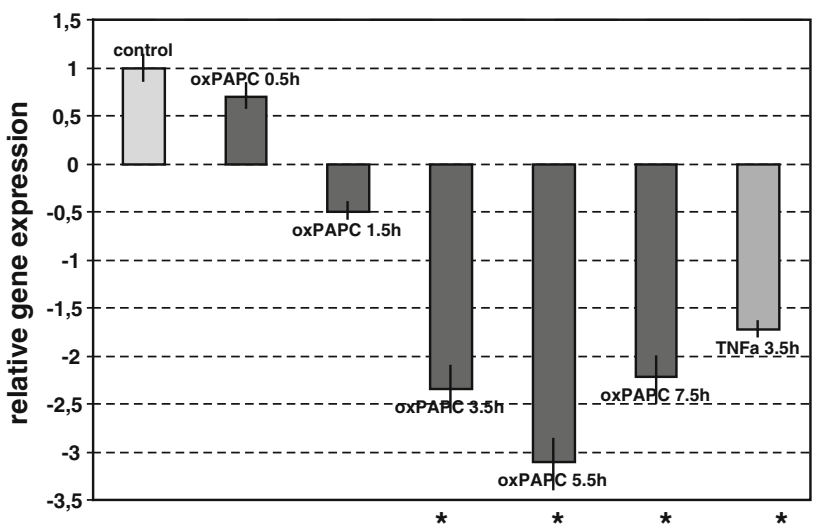

Fig. 5 The time-depended inhibition of the IL-15 gene expression in endothelial cells (HUVECs) after incubation with $150 \mu \mathrm{g} / \mathrm{ml}$ ox-PAPC. The presented results are mean values $\pm \mathrm{SEM} ; n=3$ done in triplicates $(* P<0.05)$. Control-not-stimulated HUVECs, VEGF - vascular endothelial growth factor, bFGF-basic fibroblast growth factor, ox-PAPC-oxidized 1-palmitoyl-2-archidonoyl$s n$-glycero-3-phosphocholine, TNF $\alpha$ - tumor necrosis factor alpha served as positive control of stress/inflammation condition

\section{Discussion}

Our results demonstrate pro-angiogenic IL-15 activity, which was described in some previously published observations $[6,8]$. We also verified that the pro-angiogenic

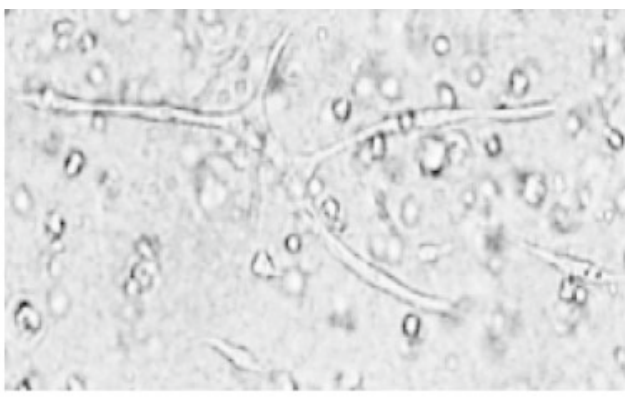

bFGF (0,5nM)

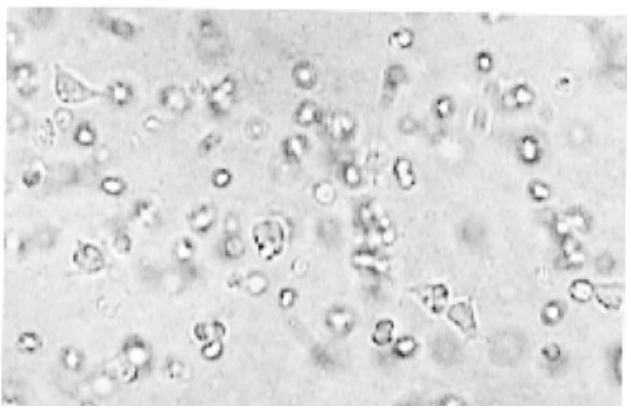

OxPAPC (150ug/ml)

Fig. 4 The influence of investigated factors on tubule-like structure formation in the 3D model. Example pictures form 3 experiments. bFGF-basic fibroblast growth factor, IL-15-interleukin 15, ox-PAPC-oxidized 1-palmitoyl-2-archidonoyl-sn-glycero-3-phosphocholine 
Fig. 6 The time-dependent influence of $150 \mu \mathrm{g} / \mathrm{ml}$ ox-PAPC on IL-8 gene expression in HUVECs. The presented results are mean values $\pm \mathrm{SEM} ; n=3$ done in triplicates $(* * P<0.005)$.

Control—not-stimulated

HUVECs, oxPAPC—oxidized 1-palmitoyl-2-archidonoylsn-glycero-3-phosphocholine, $\mathrm{TNF} \alpha$ - tumor necrosis factor alpha served as positive control of stress/inflammation condition

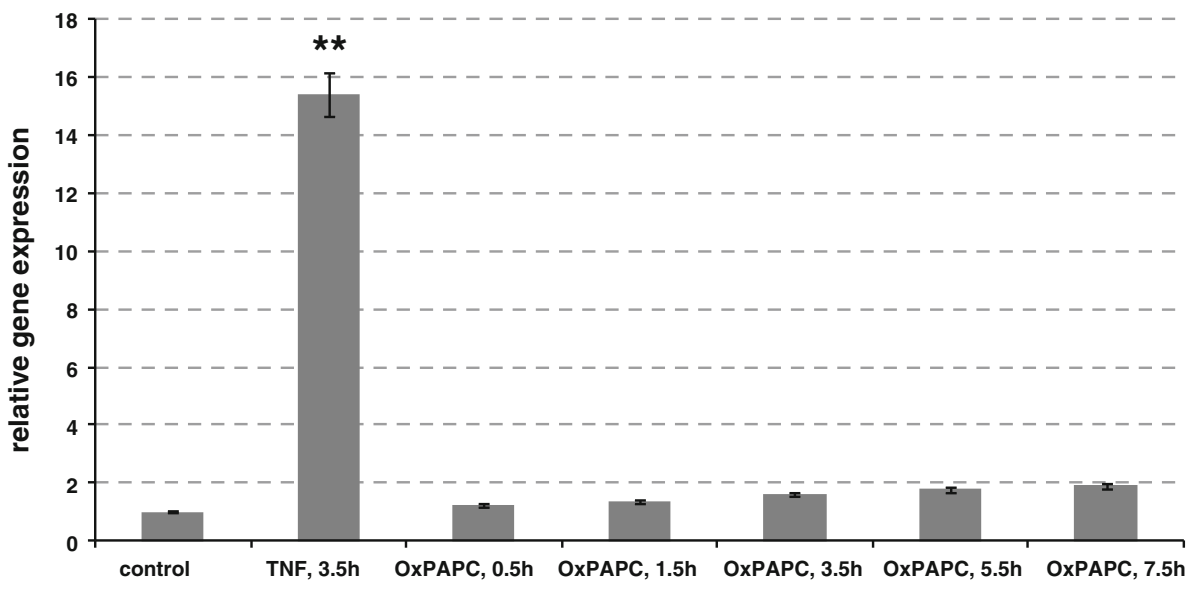

activity of IL-15 was not associated with increased proliferation of HUVECs, but it rather promoted capillary network maturation manifested by the process of the tubule formation in the 3D matrigel model. Non-toxic amounts of IL-15 increased the number and length of the endothelial tubules in a similar fashion as the pro-angiogenic VEGF and bFGF factors.

In our study, ox-PAPC did not influence the endothelial cell proliferation. Moreover, it inhibited the tubular-like structures formation in the 3D model of angiogenesis in the time-dependent way. Our in vitro observations confirmed previously reported ox-PAPC inhibitory effect on the constitutive expression of IL-15 gene in the endothelial cells [9, our results]. The inhibition of IL-15 expression mediated by ox-PAPC in HUVECs could provide some possible explanation on how breakable and immature type of capillaries form, which are typical for impaired and pathological angiogenesis associated with atherosclerotic plaque development [9].

IL-8 is a potent activator of endothelial cells chemotaxis that promotes angiogenesis [10]. Our results show weak up-regulation of IL-8 gene by ox-PAPC in HUVECs, which may also add to the understanding of the impaired angiogenesis and the atherosclerotic plaque rupture mechanism. It is possible that on the one hand ox-PAPC leads to IL-8 induction and promotes early phase of angiogenesis, while on the other hand it simultaneously down-regulates the IL-15 gene expression and inhibits maturation of the capillary network. Such a pathological mechanism might result in generation of non-functional, breakable capillaries; which typically characterize unstable atherosclerotic plaques or tumor vasculature [11].

Our findings may help understand mechanisms of vasculature formation driven by modified lipids during the pathological angiogenesis associated with the atherosclerotic plaque formation.
Acknowledgments This work was supported by Polish Committee of Science; Grant No: 4P05D 01016, and FW7 EU Grant LIPIDOMICNET No 202272. Authors thank Dr. Anna Knapp for editorial and language support during writing this manuscript.

Conflict of Interest All authors do not report conflicts of interest of this manuscript.

\section{References}

1. Martinet W, De Meyer GR (2009) Autophagy in atherosclerosis: a cell survival and death phenomenon with therapeutic potential. Circ Res 104:304-317

2. Bochkov VN, Mechtcheriakova D, Lucerna M, Huber J, Malli R, Graier WF, Hofer E, Binder BR, Leitinger N (2002) Oxidized phospholipids stimulate tissue factor expression in human endothelial cells via activation of ERK/EGR-1 and $\mathrm{Ca}(++) / \mathrm{NFAT}$. Blood 99:199-206

3. Yeh M, Gharavi NM, Choi J, Hsieh X, Reed E, Mouillesseaux KP, Cole AL, Reddy ST, Berliner JA (2004) Oxidized phospholipids increase interleukin 8 (IL-8) synthesis by activation of the c-src/signal transducers and activators of transcription (STAT)3 pathway. J Biol Chem 279:30175-30181

4. Argilés JM, López-Soriano FJ, Busquets S (2009) Therapeutic potential of interleukin-15: a myokine involved in muscle wasting and adiposity. Drug Discov Today 14:208-213

5. Fisman EZ, Adler Y, Tenenbaum A (2008) Biomarkers in cardiovascular diabetology: interleukins and matrixins. Adv Cardiol 45:44-64

6. Angiolillo AL, Kanegane H, Sgadari C, Reaman GH, Tosato G (1997) Interleukin-15 promotes angiogenesis in vivo. Biochem Biophys Res Commun 233:231-237

7. Dembinska-Kiec A, Polus A, Kiec-Wilk B, Grzybowska J, Mikolajczyk M, Hartwich J, Razny U, Szumilas K, Banas A, Bodzioch M, Stachura J, Dyduch G, Laidler P, Zagajewski J, Langman T, Schmitz G (2005) Proangiogenic activity of betacarotene is coupled with the activation of endothelial cell chemotaxis. Biochim Biophys Acta 1740:222-239

8. Kaspar M, Trachsel E, Neri D (2007) The antibody-mediated targeted delivery of interleukin-15 and GM-CSF to the tumor neovasculature inhibits tumor growth and metastasis. Cancer Res 67:4940-4948 
9. Houtkamp MA, van Der Wal AC, de Boer OJ, van Der Loos CM, de Boer PA, Moorman AF, Becker AE (2001) Interleukin-15 expression in atherosclerotic plaques: an alternative pathway for T-cell activation in atherosclerosis? Arterioscler Thromb Vasc Biol 21:1208-1213

10. Heidemann J, Ogawa H, Dwinell MB, Rafiee P, Maaser C, Gockel HR, Otterson MF, Ota DM, Lugering N, Domschke W, Binion DG (2003) Angiogenic effects of interleukin 8 (CXCL8) in human intestinal microvascular endothelial cells are mediated by CXCR2. J Biol Chem 278:8508-8515

11. Miyamoto S, Yagi H, Yotsumoto F, Horiuchi S, Yoshizato T, Kawarabayashi T, Kuroki M, Mekada E (2007) New approach to cancer therapy: heparin binding-epidermal growth factor-like growth factor as a novel targeting molecule. Anticancer Res $27: 3713-3721$ 